\title{
Novel combined variants of WT1 and TET2 in a refractory and recurrent $A M L$ patient
}

Qiang Ma, Yixian Guo, Xiaoxi Lan, Guoxiang Wang and Wanling Sun*

\begin{abstract}
Background: Somatic mutations in Wilms' tumor 1 (WT1) and tet methylcytosine dioxygenase 2 (TET2) genes were separately perceived as contributors to hematopoietic disorders and usually thought to have a mutually exclusive effect in acute myeloid leukemia (AML). However, we found novel WT1 and TET2 variants persistently co-existed in a refractory and recurrent AML patient with $\mathrm{t}(9 ; 11)(\mathrm{p} 21.3 ; \mathrm{q} 23.3) ; K M T 2 A-M L L T 3$, and were only detectable genetic alteration in early recurrence. Hence, these two novel variants were further investigated in patient's family, and the potential effect on disease progression was evaluated at follow-up.

Case presentation: A 27-year-old male was diagnosed with AML, having t(9;11)(p21.3;q23.3); KMT2A-MLLT3, accompanied by WT1 (NM_024426.6:exon7:c.1109G >C:p.Arg370Pro) and TET2 (NM_001127208.3:exon11:c.5530G>A:p. Asp1844Asn) variants. After two cycles of induction chemotherapy, complete remission was achieved. A consolidation treatment was then completed. However, the evaluation of the bone marrow revealed that early recurrence, WT1 (p.Arg370Pro) and TET2 (p.Asp1844Asn) variants still detectable, instead of KMT2A-MLLT3. Subsequently, these two variants were proved to be germline variants, which inherited from father and mother respectively. And the patient's elder brother also carried TET2 (p.Asp1844Asn) variant. A sequential allogeneic HLA-matched sible hematopoietic stem cell transplantation (allo-HSCT) was carried out, and the donor is the patient's elder brother, the original two variants of patient were replaced by the donor-derived TET2 (p.Asp1844Asn) variant after allo-HSCT; the patient has remained in complete remission with regular follow-up.
\end{abstract}

Conclusions: In brief, it is firstly reported that WT1 p.Arg370Pro and TET2 p.Asp1844Asn variants co-existed in a refractory and recurrent AML patient by inheritance. These two variants of the patient were replaced with donorderived TET2 p.Asp1844Asn after allo-HSCT, and the patient has remained in complete remission with regular follow-up.

Keywords: Acute myeloid leukemia, WT1, TET2, Germline variant, Next-generation sequencing, Prognosis, Case report

\section{Background}

Acute myeloid leukemia (AML) is a clonal hematopoietic disorder and is usually observed along with genetic alterations in hematopoietic stem cells [1]. Most of the molecular cytogenetic abnormalities involved in AML have been identified, and these may be potential biomarkers

*Correspondence: wanlingsun@xwhosp.org

Department of Hematology, Xuanwu Hospital, Capital Medical University,

No. 45 Changchun Street, Beijing 100053, People's Republic of China for differential diagnosis, risk stratification, and therapeutic response in AML patients [2, 3]. Nevertheless, genetic alterations with definite clinical significance are relatively rare [4], and the clinical significance of most of them is still unknown [5], especially for germline mutations [6].

In our hospital, a 27-year-old male was diagnosed with AML, having $\mathrm{t}(9 ; 11)(\mathrm{p} 21.3 ; \mathrm{q} 23.3) ; \quad K M T 2 A-$ MLLT3, accompanied by Wilms' tumor 1 (WT1, NM_024426.6:exon7:c.1109G>C:p.Arg370Pro) and tet 


\section{(See figure on next page.)}

Fig. 1 Representative results of laboratory tests of the patient with AML. a Monoblasts were observed in bone marrow of the patient, and some had very abundant cytoplasm(OLYMPUS BX53; Smart Digital Camrea;original magnification, $\times 1000)$; b, c P4 stands for abnormal cells, accounted for 96\%. HLA-DR(+), CD33(+), CD15(+), CD64(+), CD38(+), CD123(partly+), CD56(+);CD11b(partly+), CD34(partly+), CD117(partly+); CD10(-), CD19(-), CD20(-), MPO(-), CCD3(-), CCD79a(-), CD13(-), CD7(-), CD5(-), CD14(-). d Chromosome karyotype analysis showed that the patient with 46, XY, $t(9 ; 11)(p 21.3 ; q 23.3)$. e Complete donor chimerism was observed after allo-HSCT by short tandem repeat polymerase chain reaction (STR-PCR) analysis

methylcytosine dioxygenase 2 (TET2, NM_001127208. 3:exon11:c.5530G>A:p.Asp1844Asn) variants, without abnormal WT1 mRNA expression. However, there are some noteworthy issues in this case, for instance: Why did WT1 and TET2 variants, which were previously reported to be mutually exclusive $[7,8]$, persistently coexist in the AML patient? WT1 (p.Arg370Pro) and TET2 (p.Asp1844Asn) variants were only detectable genetic alterations in patients with early recurrence, instead of $K M T 2 A-M L L T 3$, and whether co-exist of these two variants contribute to disease progression? To address these issues, blood and buccal mucosa cells samples of patient and his family were firstly collected, targeted multi-genes panel sequencing was performed to determine whether variation is hereditary or acquired. And after allo-HSCT, targeted multi-genes panel sequencing was performed again to investigate whether the variants were replaced, and regularly follow-up was performed.

\section{Case presentation}

A 27-year-old male was admitted to our hospital in Jan 2019 due to hypoleukocytosis lasting two weeks. His family history did not suggest an inherited susceptibility to cancer, as no first-degree relatives, including his parents, his brother, and his child, had cancer. The complete blood cell count $(\mathrm{CBC})$ indicated a white blood cell (WBC) count of $0.77 \times 10^{9} / \mathrm{L}$, platelet count of $129 \times 10^{9} / \mathrm{L}$, and hemoglobin $(\mathrm{Hb})$ concentration of $45 \mathrm{~g} / \mathrm{L}$. Bone marrow aspiration revealed monocyte hyperplasia with $93 \%$ monoblasts and $2.5 \%$ promonocytes (Fig. 1a). Moreover, non-specific esterase stain revealed intensely positive monoblasts, and the positive stain could be inhibited by sodium fluoride (data not shown). Immunophenotype analysis of the abnormal cells by flow cytometry revealed full expression of HLA-DR, CD38, CD33, CD15, CD64 and CD56; partial expression of CD34, CD117, CD123, CD11b; and no expression of CD13, CD7, CD5, CD19, CD10, CD20, CD14, MPO, cCD79a and cCD3 (Fig. 1b, c). Chromosome analysis of bone marrow cells indicated a karyotype of 46, XY, t(9;11)(p21.3;q23.3) (Fig. 1d). The KMT2A-MLLT3 fusion gene was identified using qRT-PCR.Targeted multi-genes panel sequencing revealed that WT1 (p.Arg370Pro, VAF 49.19\%) and TET2 (p.Asp1844Asn, VAF 25.25\%) variants were present. He was diagnosed with AML, having $\mathrm{t}(9 ; 11)(\mathrm{p} 21.3 ; \mathrm{q} 23.3)$, KMT2A-MLLT3, accompanied by WT1 and TET2 variants, intermediate risk. A standard IA induction chemotherapy regimen (idarubicin + cytarabine) was administered from January 30, 2019.

At the end of first induction chemotherapy on February 20,2019 , the CBC revealed a WBC count of $0.64 \times 10^{9} / \mathrm{L}$, $\mathrm{Hb}$ of $83 \mathrm{~g} / \mathrm{L}$, platelet count of $21 \times 10^{9} / \mathrm{L}$. Bone marrow aspiration showed that primitive and immature cells made up $6 \%$ of the nucleated bone marrow cells. Flow cytometry detected $21 \%$ abnormal immunophenotype monocytes. The patient achieved partial remission. The CAG (Clarithromycin + Cytarabine + G-CSF) regimen was then administered as the second induction chemotherapy from February 21, 2019.

The evaluation of the bone marrow on March 21, 2019, showed that primitive and immature monocytes made up $0 \%$ of the bone marrow nucleated cells. Monocytes with abnormal immunophenotype were less than $0.01 \%$. The KMT2A-MLLT3 fusion gene was not present. Complete remission was achieved. However, the WT1 (p.Arg370Pro VAF 50.11\%) and TET2 (p.Asp1844Asn VAF $48.33 \%$ ) variants were still present. Another IA regimen was then administered as consolidation chemotherapy from March 29, 2019.

The evaluation of the bone marrow on May 9, 2019 revealed that primitive and immature monocytes made up 7\% of the nucleated bone marrow cells and CD34+ CD $117+$ CD33+ cells were $0.75 \%$ by flow cytometry. KMT2A-MLLT3 fusion remained negative. All these data suggested early recurrence. While WT1 (p.Arg370Pro) and TET2 (p.Asp1844Asn) variants were still positive, the VAF was $51.87 \%$ and $51.78 \%$, respectively.

Owing to the fluctuation of the disease, the risk stratification was re-evaluated to high-risk. A HA regimen chemotherapy (homoharringtonine and intermediatedose cytarabine) was administered from May 11, 2019. The second complete remission was achieved, with variants on WT1 (p.Arg370Pro) and TET2 (p.Asp1844Asn) genes still present, and the VAF was $48.35 \%$ and $49.88 \%$, respectively (Table 1 ).

A sequential allogeneic HLA-matched sible hematopoietic stem cell transplantation was carried out on July 9, 2019, and the donor is patient's elder brother. After alloHSCT, complete donor chimerism was received (Fig. 1e), 
a

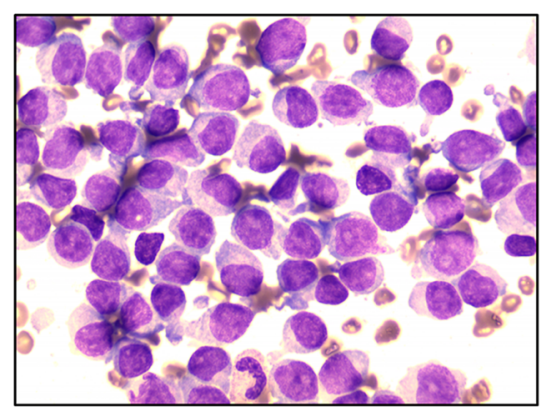

c
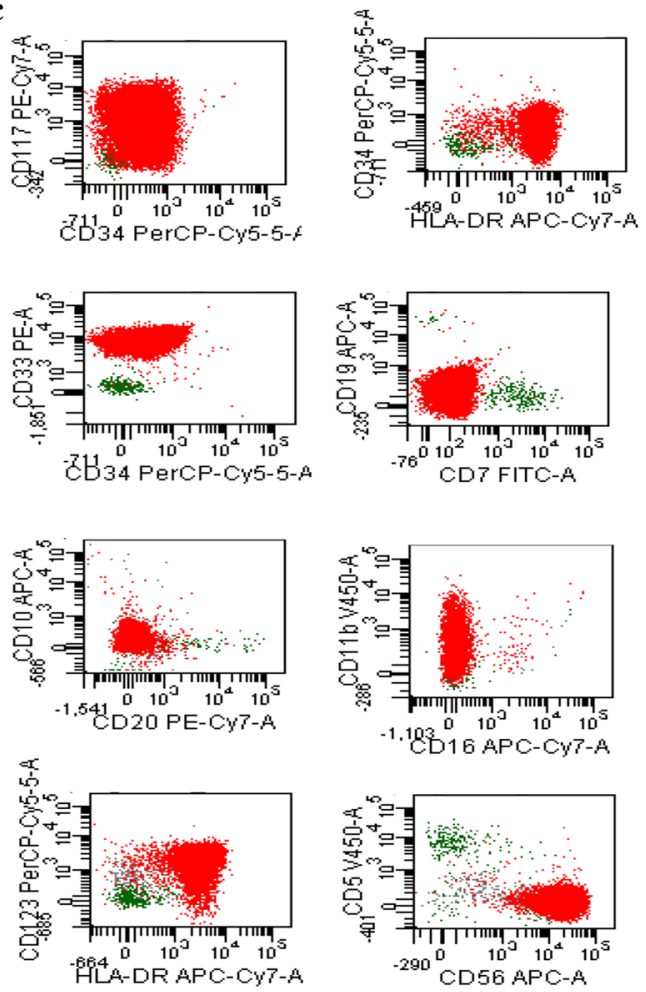
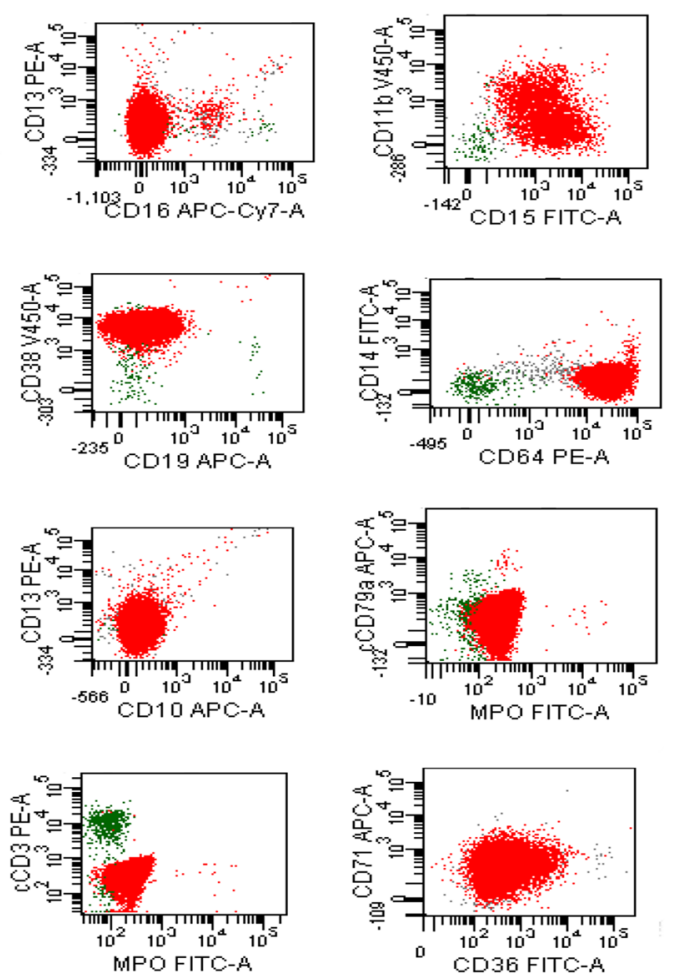

d

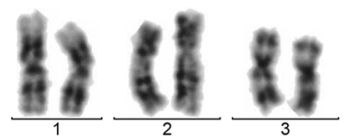

III

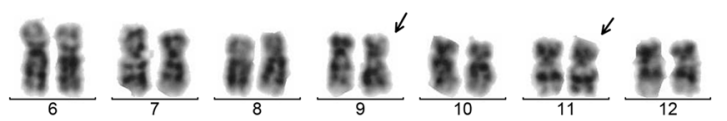

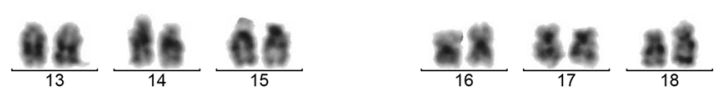

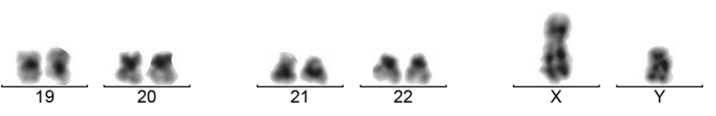

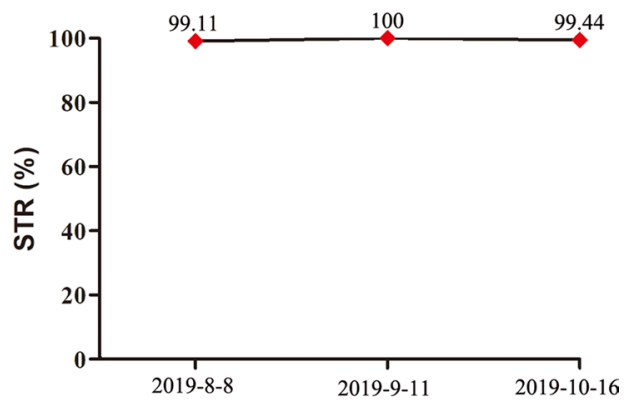


Table 1 Molecular and cellular genetic alternation of patient at different disease stages

\begin{tabular}{|c|c|c|c|c|c|c|c|}
\hline \multirow[t]{2}{*}{ Examination } & \multicolumn{4}{|l|}{ Before allo-HSCT } & \multicolumn{3}{|c|}{ After allo-HSCT } \\
\hline & Initial diagnosis & CR1 & Relapse & CR2 & 1 & 2 & 3 \\
\hline Karyotype & $\mathrm{t}(9 ; 11)(\mathrm{p} 21.3 ; \mathrm{q} 23.3)$ & NA & - & NA & - & - & NA \\
\hline Fusion & KMT2A-MLLT3 & - & - & NA & - & NA & NA \\
\hline \multirow[t]{2}{*}{ Variants (VAF \%) } & TET2 (25.25) & TET2 (48.33) & TET2 (51.78) & TET2 (49.88) & TET2 (48.58) & TET2 (50.20) & TET2 (52.59) \\
\hline & WT1 (49.19) & WT1 (50.11) & WT1 (51.87) & WT1 (48.35) & & & \\
\hline WT1 level (\%) & 0 & NA & 0.03 & 0.15 & NA & NA & 0 \\
\hline
\end{tabular}

- Normal karyotype or fusion gene negative; allo-HSCT Allogeneic hematopoietic stem cell transplantation, NA Not available, CR1 complete remission after two cylces inductive chemotherapy, CR2 complete remission after one cycle reinductive chemotherapy; WT1 level (\%) $=($ WT1 mRNA copies/ABL1 mRNA copies) $\times 100$, reference range of WT1 mRNA level in our laboratory is 0-0.6\%.WT1:NM_024426.6:exon7:c.1109G>C:p.Arg370Pro; TET2:NM_001127208.3:exon11:c.5530G>A:p.Asp1844Asn

\begin{tabular}{|l|l|l|l|l|l|}
\hline WT1 (p.Arg370Pro) & Gene & Individual & Transcript & $\begin{array}{c}\text { Coding and Amino } \\
\text { Acid Change }\end{array}$ \\
\hline & & & Father & NM_024426 & c.1109G>C p.R370P \\
\hline
\end{tabular}

and donor-derived TET2 p.Asp1844Asn variant was also received. To date, the patient has remained in complete remission with regular follow-up.

Since the patient's variants were persistent and does not change with treatment, we suspected the WT1 (p.Arg370Pro) and TET2 (p.Asp1844Asn) variants were germline variants. Then buccal mucosa cells (somatic control) were first obtained from the patient for targeted sequencing, and results revealed that shared variation sites of WT1 p.Arg370Pro (52.09\%) and TET2 p.Asp1844Asn (37.26\%) existed in buccal mucosa cells, indicating that WT1 p.Arg370Pro and TET2 p.Asp1844Asn may germline variants.

To further identify whether these two variants were heritable, DNA samples from the patient's relatives were analyzed using the same target sequencing; WT1 p.Arg370Pro (VAF 44.75\%) was detected in the patient's father and TET2 p.Asp1844Asn (VAF 36.01\%) was detected in his mother; the brother carried the TET2 p.Asp1844Asn variant (VAF 48.30\%) (Fig. 2). The above results thoroughly confirmed that germline variants WT1 p.Arg370Pro and TET2 p.Asp1844Asn co-existed in the patient by inheritance.

\section{Discussion and conclusions}

In this study, we first reported that WT1 p.Arg370Pro co-exists with TET2 p.Asp1844Asn variant in a refractory and recurrent AML patient. These two variants were derived from the patient's father and mother respectively, and were only detectable genetic alteration in early recurrence. These two variants were replaced with donorderived TET2 p.Asp1844Asn variant after allo-HSCT, and the patient has remained in complete remission with regular follow-up.

WT1 and TET2 mutations were mutually exclusive in AML patient, it is rare that WT1 and TET2 variants co-exist in an AML patient [8]. As the co-existing variants in this case were germline variants derived from the patient's father and mother, respectively, this case serves as a reminder that mutually exclusive germline variants may co-exist in a patient due to inheritance. Recently, a growing number of germline mutations associated with tumor susceptibility have been reported; for instance, MITF (Microphthalmia-associated transcription factor) mutation impairs SUMOylation, which has an oncogenic function in the tumorigenesis of multiple tissues/melanocytes and kidney cells [9]. GATA2, RUNX1, DDX41, 
PAX5, CEBPA, and TP53 are associated with hematologic malignancies predisposition [10]. Identifying germline mutations is beneficial for therapy choice, donor selection for hematopoietic stem cell transplantation, evaluation of comorbidities, and surveillance strategies to improve the clinical outcomes [10].

Although it is unclear whether co-exist of these two variants closely associated with AML predisposition and recurrence, WT1 and TET2 genes are necessary for the normal development of blood cells based on previous findings. TET2 is important for normal myelopoiesis, and disruption of TET2 enzymatic activity favors myeloid tumorigenesis [11]. Slower proliferation and reduced clonogenicity have been observed in TET2 overexpressing cells in vitro compared to those transfected with the empty vector, which indicates the suppressor role of TET2 in AML. However, the inhibitory effects of TET2 on leukemic cell proliferation and colony formation could be abolished by WT1 knockdown [11]. Further studies revealed that TET2 suppresses leukemia cell proliferation and colony formation in a WT1-dependent manner [12]. All of the above conclusions are from in vitro and animal experiments; here, for the first time, TET2 and WT1 variants are reported to co-exist in an AML patient, with detectable alterations only in the complete remission and relapse stages. Interesting, when these two variants were replaced with donor-derived TET2 p.Asp1844Asn variant, patient has been received complete remission during follow-up. It is necessary to explored whether co-exist of WT1 and TET2 variants have a worse effect on disease progression compared with existed alone.

Since the interaction of these two genes is essential for biological functions, we attempted to explain the pathogenesis at the molecular level. TET2 binds to the zinc finger domain (residue 323-449), but not the $\mathrm{N}$-terminal region (residue 1-323) of $W T 1$. In this patient, the missense mutation was in the zinc finger domain (residue 370) of WT1, which may disrupt the binding between WT1 and TET2. Moreover, WT1 binds to the CD domain of TET2, but not the $\mathrm{N}$-terminal region (residue 1 to 1127) [11]. Unfortunately, in this case, the missense mutation was in the CD domain (residue 1844) of TET2, which may further disrupt the binding between WT1 and TET2. From a molecular point of view, this may be a reasonable explanation for the disease. In clinical practice, WT1 expression and disease progression are usually parallel to each other; WT1 expression is also used as the MRD marker of hematological neoplasms when other genetic alterations are absent [13]. However, in this case, abnormal up-regulation of WT1 was not observed at different stages, but the $W T 1$ variant persisted. Similar to the $W T 1$ mutation being related to poor prognosis [14], WT1 overexpression was also indicated to be associated with shorter OS [15]. However, the question remains, which alteration could be a potential indicator of prognostic stratification for the patient; expression level or mutation status? It seems contradictory for the patient to have WT1 variant and no WT1 overexpression, and related reports in the literature are limited.

In this case, WT1 and TET2 variants were the only molecular alterations in the relapse stage. Then, this is only a phenomenon we observed, and the existing evidence is not enough to show that co-existing WT1 and TET2 variants were involved in the occurrence and progression of AML in the above manner. Previous studies have revealed that $W T 1$ can bind TET2, acting as a guide for TET2 to specific genomic loci, leading to increased stem cell function and self-renewal with proliferation [16]. This seems a reasonable explanation for why the patient relapsed in a short time, but other possible reasons also need to be further explored. However, after allo-HSCT, two variants of patient were replaced with donor-derived TET2 p.Asp1844Asn, then patient has remained in complete remission with regular followup, we have to speculate whether there was the possibility of concurrent presence of WT1 gene mutation could contribute to second hit in the pathogenesis of AML. In brief, these two variants might play some role in the pathogenesis of refractory and recurrent AML, while a long term follow-up and regular monitoring by NGS is needed. At the same time, we just report a case and the result of the family pedigree study. More cases and related families, and further researches are necessary to prove our hypothesis.

In summary, we present the first report that $W T 1$ and TET2 variants co-existed in a refractory and recurrent AML patient; these were identified as germline variants. After allo-HSCT, the original variants were replaced by the donor-derived TET2 variant; the patient has remained in complete remission with regular follow-up.

\section{Abbreviations \\ AML: Acute myeloid leukemia; TET2: Tet methylcytosine dioxygenase 2; WT1: Wilms'tumor 1; MRD: Minimal residual disease; VAF: Variant allele frequency; CBC: Complete blood cell count; WBC: White blood cell count; Hb: Hemo- globin; HSCT: Hematopoietic stem cell transplantation; qRT-PCR: Quantitative real-time PCR; OS: Overall survival.}

\section{Acknowledgements}

Not applicable.

\section{Authors' contributions}

QM/YXG wrote the manuscript. QM/XXL/GXW collected samples and performed data analysis. WLS designed this study, critically reviewed the manuscript. All authors have read and approved the manuscript.

\section{Funding}

Supported by Beijing Natural Science Foundation (No.Z200022), and Xuanwu Hospital Foundation, Capital Medical University (No. XWJL-2019023). The 
funding body did not have any role in the design of the study and collection, analysis, and interpretation of data and in writing the manuscript.

\section{Availability of data and materials}

The patient's DNA sequencing data generated during the current study are deposited in the NCBI Sequence Read Archive (SRA) repository under the accession number PRJNA729676. The datasets generated and/or analyzed during the current study are available in the Genbank repository (GRCh37/hg19, https://www.ncbi.nlm.nih.gov/genome/guide/human/) for NM_024426.6 (https://www.ncbi.nlm.nih.gov/nuccore/NM_024426.6/) and NM_001127208.3 (https://www.ncbi.nlm.nih.gov/nuccore/NM_001127208.3). The raw datasets of family members generated during the current study are not publicly available because it is possible that individual privacy could be compromised. It is possible to apply for permission to obtain access to the raw sequencing data through the corresponding author.

\section{Declarations}

\section{Ethics approval and consent to participate}

The present study was permitted by Ethics Committee of Xuanwu Hospital, Capital Medical University, and written informed consents were obtained from all of the participants.

\section{Consent for publication}

Written informed consent for publication of identifying images or other personal or clinical details was obtained from all of the participants. A copy of the written consent is available for review by the Editor of this journal.

\section{Competing interests}

The authors declare that they have no competing interests.

Received: 24 September 2020 Accepted: 7 June 2021

Published online: 13 June 2021

\section{References}

1. Allahyari A, Tajeri T, Sadeghi M. Prognostic factors and survival in acute myeloid leukemia cases: a report from the Northeast of Iran. Asian Pac J Cancer Prev. 2016;17:1547-51.

2. Iwabuchi S, Fukui S. [Post-transfusion acute non-A, non-B hepatitis]. Sugai J Nihon rinsho Jpn J Clin Med. 1988:46:2663-8.
3. Vosberg S, Greif PA. Clonal evolution of acute myeloid leukemia from diagnosis to relapse. Genes Chromosomes Cancer. 2019;6:66.

4. Wang M, Yang C, Zhang L, Schaar DG. Molecular mutations and their cooccurrences in cytogenetically normal acute myeloid leukemia. Stem Cells Int. 2017:2017:6962379.

5. Hirsch P, Qassa G, Marzac C, et al. Acute myeloid leukemia in patients older than 75: prognostic impact of FLT3-ITD and NPM1 mutations. Leuk Lymphoma. 2015;56:147-50.

6. Maciejewski JP, Padgett RA, Brown AL, Muller-Tidow C. DDX41-related myeloid neoplasia. Semin Hematol. 2017;54:94-7.

7. Du D, Zhu L, Wang Y, Ye X. Expression of WT1 gene and its prognostic value in patients with acute myeloid leukemia. Zhejiang da xue xue bao Yi xue ban J Zhejiang Univ Med Sci. 2019;48:50-7.

8. Bowman RL, Levine RL. TET2 in normal and malignant hematopoiesis. Cold Spring Harbor Perspect Med. 2017;7:66.

9. Paillerets BB, Lesueur F, Bertolotto C. A germline oncogenic MITF mutation and tumor susceptibility. Eur J Cell Biol. 2014;93:71-5.

10. Furutani E, Shimamura A. Germline genetic predisposition to hematologic malignancy. J Clin Oncol. 2017;35:1018-28.

11. Ko M, Huang $Y$, Jankowska AM, et al. Impaired hydroxylation of 5-methylcytosine in myeloid cancers with mutant TET2. Nature. 2010;468:839-43.

12. Wang $Y$, Xiao $M, C$ Chen $X$, et al. WT1 recruits TET2 to regulate its target gene expression and suppress leukemia cell proliferation. Mol Cell. 2015:57:662-73.

13. Rossi G, Minervini MM, Carella AM, Melillo L, Cascavilla N. Wilms'Tumor Gene (WT1) Expression and minimal residual disease in acute myeloid leukemia. In: van den Heuvel-Eibrink MM ed. Wilms tumor. Brisbane; 2016.

14. Niktoreh N, Walter C, Zimmermann M, et al. Mutated WT1, FLT3-ITD, and NUP98-NSD1 fusion in various combinations define a poor prognostic group in pediatric acute myeloid leukemia. J Oncol. 2019;2019:1609128.

15. Liu H, Wang $X$, Zhang $H$, et al. Dynamic changes in the level of WT1 as an MRD marker to predict the therapeutic outcome of patients with AML with and without allogeneic stem cell transplantation. Mol Med Rep. 2019;20:2426-32.

16. Pronier E, Bowman RL, Ahn J, et al. Genetic and epigenetic evolution as a contributor to WT1-mutant leukemogenesis. Blood. 2018;132:1265-78.

\section{Publisher's Note}

Springer Nature remains neutral with regard to jurisdictional claims in published maps and institutional affiliations.
Ready to submit your research? Choose BMC and benefit from:

- fast, convenient online submission

- thorough peer review by experienced researchers in your field

- rapid publication on acceptance

- support for research data, including large and complex data types

- gold Open Access which fosters wider collaboration and increased citations

- maximum visibility for your research: over $100 \mathrm{M}$ website views per year

At BMC, research is always in progress.

Learn more biomedcentral.com/submissions 